Como citar este artigo: TAVARES, DEISE I.: KURTZ. MANUELLA C.; SCHLEMMER, CESSICA B. V.; SOUZA, CORA C.; DOS SANTOS, TAMIRES D.: VENDRUSCULO, ALECSANDRA P.; PIVETTA, HEDIONEIA MARIA F. BRAZ, MELISSA M.; Caracterização das pacientes com incontinência urinária atendidas em um serviço de fisioterapia ambulatorial. Revista Saúde (Sta. Maria). 2020; $46(1)$.

\begin{abstract}
Autor correspondente: Nome: Deise lop Tavares E-mail: deiseiop@hotmail.com Telefone: (55) 98406-3131 Formação Profissional: : Formada em Fisioterapia pela Universidade Franciscana (UFN) que fica na cidade de Santa Maria, Rio Grande do Sul, Brasil. Especialista em Reabilitação Físico Motora pela Universidade Federal de Santa Maria (UFSM), Mestranda em Cerontologia pela UFSM.
\end{abstract}

Filiação Institucional: Universidade Federal de Santa Maria (UFSM) Endereço para correspondência: Rua: Avenida Roraima no 1000 , Sala 4 108, prédio 26D, Centro de Ciências da Saúde

Bairro: Camobi

Cidade: Santa Maria

Estado: Rio Grande do Sul

CEP: 97 105-900

Data de Submissão:

03/02/2020

Data de aceite:

26/03/2020

Conflito de Interesse: Não há conflito de interesse

\section{Caracterização das pacientes com incontinência urinária atendidas em um serviço de fisioterapia ambulatorial}

\section{Characterization of patients with urinary incontinence treated at an outpatient physiotherapy service}

Deise lop Tavares, Manuella Chagas Kurtz, Gessica Bordin Viera Schlemmer, Cora da Gama Souza, Tamires Daros dos Santos, Alecsandra Pinheiro Vendrusculo, Hedioneia Maria Foletto Pivetta, Melissa Medeiros Braz

\section{RESUMO}

Introdução: A Incontinência urinária é considerada uma das novas epidemias associada com aumento da sobrevida, sendo mais frequente nas mulheres. Objetivo: Caracterizar as pacientes com incontinência urinária que foram atendidas no serviço de Fisioterapia de um serviço secundário de média complexidade no período de 2015 a 2017. Métodos: Estudo transversal, descritivo, quantitativo realizado a partir de uma análise documental de prontuários de Fisioterapia de um serviço secundário de média complexidade durante o período de 2015 a 2017. Resultados: A IU esteve presente em mulheres com média de idade de 58,2 anos, casadas, com hipertensão ou constipação associadas, não praticavam atividade física, com prevalência de parto vaginal e com episiotomia, As perdas ocorreram mais durante o dia, em forma de jato, ao esforço e após a tosse ou espirros. Conclusão: Espera-se que com estes dados se tenha um maior conhecimento sobre a incontinência urinária e seus agravos e se possam elaborar ações para desmistifica-las.

PALAVRAS-CHAVE: Incontinência urinária; Incontinência urinária de urgência; Incontinência urinária por estresse; Fisioterapia.

\section{ABSTRACT}

Objective: Introduction: Urinary incontinence is considered one of the new epidemics associated with increased survival, being more frequent in women. Objective: To characterize the patients with urinary incontinence who were treated in the Physiotherapy service of a secondary service of medium complexity in the period from 2015 to 2017 . Methods: A cross-sectional, descriptive and quantitative study was carried out based on a documentary analysis of medical records of one secondary service of medium complexity during the period from 2015 to 2017. Results: UI was present in women with a mean age of 58.2 years, married, with associated hypertension or constipation, did not practice physical activity, with a prevalence of vaginal delivery and with episiotomy, losses occurred more during the day, in jet form, at exertion and after coughing or sneezing. Conclusion: It is expected that with these data a greater knowledge about the urinary incontinence and its aggravations will be able to be elaborated and actions to demystify them.

KEYWORDS: Urinary Incontinence; Urinary Incontinence, Urge; Urinary Incontinence, Stress; Physical Therapy Specialty. 


\section{INTRODUÇÃO}

A incontinência Urinária (IU) é definida como qualquer perda involuntária de urina. Pode ser classificada em Incontinência Urinária de Esforço, Incontinência Urinária de Urgência e Incontinência Urinária Mista1'. É considerada um problema de saúde pública, pois acomete populações de diversos países e sua prevalência tem aumentado com 0 envelhecimento populacional².

O aparecimento da IU tem origem multifatorial onde o envelhecimento, aspectos genéticos, gravidez, parto, obesidade e histerectomia, são os principais fatores. É considerada uma das novas epidemias do século XXI, associada com aumento da sobrevida, sendo mais frequente nas mulheres ${ }^{1}$. Segundo o Ministério da Saúde, a IU afeta milhões de indivíduos em todas as idades. Contudo, manifesta-se com maior frequência em mulheres na fase da perimenopausa. Estudos descrevem que prevalência média de IU de 27,6\% em mulheres, e 10,5\% em homens.

Nas mulheres, a IU está relacionada a fatores de risco como a redução da pressão máxima de fechamento uretral, diminuição da vascularização, atrofia dos tecidos que revestem e envolvem a uretra, a bexiga e a vagina. Ainda, incluise os fatores anatômicos, envelhecimento natural das fibras musculares do assoalho pélvico, obesidade, antecedentes obstétricos e a menopausa ${ }^{3}$.

A IU causa muitas limitações, tanto na vida social, emocional, como também na vida sexual, o que pode levar ao isolamento social e até mesmo a depressão. Em virtude disso, reflete diretamente no bem-estar e na qualidade de vida do indivíduo ${ }^{3}$. O diagnóstico correto é importante na avaliação e no tratamento das mulheres, assim como, a determinação do efeito da qualidade de vida das pacientes ${ }^{3}$.

Existem vários tipos de tratamento para a IU, sendo os mais usados o tratamento cirúrgico, medicamentoso e fisioterapêutico. A fisioterapia tem procurando se integrar aos programas já desenvolvidos, sendo eficaz para promoção da saúde, no tratamento e redução das incapacidades, priorizando a melhora da qualidade de vida 4 .

Justifica-se a importância deste estudo devido à alta prevalência da IU em mulheres bem como a interferência que provoca na qualidade de vida das pacientes bem como na busca por tratamentos adequados. Ainda, estudos que englobam a caracterização tornam-se importantes pois podem refletir a realidade de outros serviços bem como impactar para uma melhor prestação destes serviços. Diante disso, este estudo tem como objetivo caracterizar as pacientes com incontinência urinária que foram atendidas no serviço de Fisioterapia de um serviço secundário de média complexidade no período de 2015 a 2017.

\section{MÉTODO}

Estudo transversal, descritivo, quantitativo realizado a partir de uma análise documental de prontuários de Fisioterapia de um serviço secundário de média complexidade de Santa Maria, Rio Grande do Sul, realizadas durante 0 
período de 2015 a 2017.

Como critérios de inclusão para este estudo foram as mulheres atendidas no setor de Fisioterapia pelos acadêmicos da Universidade Federal de Santa Maria (UFSM), ter procurado o atendimento devido a incontinência urinária e ter recebido alta do atendimento fisioterápico. Foram excluídas aquelas mulheres que não solicitaram atendimento devido a IU e também aquelas que ainda estavam em tratamento.

Foram analisados 56 prontuários de pacientes atendidas no setor de Fisioterapia. Nestes prontuários continha os dados sociodemográficos como idade e estado civil, queixa principal, comorbidades, cirurgias, medicamentos utilizados, hábitos como fumar e beber, dados ginecológicos como menopausa, reposição hormonal, menstruação, dados obstétricos, atividade física, atividade sexual, hábitos urinários tais como tempo de IU, caráter, incontinência, uso e tipo de proteção, situações de perdas urinárias por esforço e perda urinária em casos como stress, frio, barulho de água corrente e mãos na água.

Os dados coletados foram organizados em uma planilha idealizada para essa finalidade, usando o programa Excel e os resultados foram expressos pela estatística descritiva em frequência absoluta e relativa, sendo apresentados por meio de tabelas e figuras. Este estudo faz parte de um projeto maior intitulado "Saúde e funcionalidade no envelhecimento humano" e tem a aprovação pelo Comitê de Ética em Pesquisa da instituição responsável (Número 3.074.107 CAEE: 03467718.5.0000.5346).

\section{RESULTADOS}

Dos 56 prontuários investigados, observou-se que as mulheres atendidas tinham de 35 a 78 anos, com uma média de idade de 58,2 \pm 10,52 anos. Em relação ao estado civil, 38 mulheres se declararam casadas, oito eram divorciadas, duas solteiras, duas viúvas e seis não informaram.

No que diz respeito às queixas principais que fizeram buscar o atendimento fisioterapêutico, $100 \%$ foram por IU. A IU por esforço esteve presente em treze casos, a IU mista esteve presente em nove mulheres e a IU de urgência este presente em dois casos. A IU esteve associada com a incontinência fecal em três casos e com prolapso em oito casos. Nos demais casos (21) não foi apresentada o tipo de IU. Os problemas associados são descritos na tabela 1.

Tabela 1: Caracterização dos problemas associados da amostra.

\begin{tabular}{lc}
\hline Problemas associados & $\mathbf{n}$ \\
\hline Hipertensão & 16 \\
Constipação & 16 \\
Hemorroidas & 11 \\
Diabetes & 10
\end{tabular}


$\begin{array}{ll}\text { Doenças respiratórias } & 09\end{array}$

$\begin{array}{ll}\text { Prolapso } & 08\end{array}$

$\begin{array}{ll}\text { Infecção urinária } & 08\end{array}$

$\begin{array}{ll}\text { Dificuldade de conter gases } & 06\end{array}$

$\begin{array}{ll}\text { Obesidade } & 04\end{array}$

Hipercolesterolemia $\quad 04$

$\begin{array}{ll}\text { Artrose } & 04\end{array}$

Hipotireoidismo 03

Arritmia, depressão, disfunção cardíaca, hérnia de disco, gastrite, osteopenia, 02

disfunção renal e rinite, respectivamente.

Diverticulite, diarreia, angina, dor pélvica, labirintite, Parkinson, quedas,

Síndrome de Reynaud, poliomiosite, distúrbios circulatórios, problemas na

coluna, ansiedade, glaucoma, artrite reumatoide, osteoporose, ovário policístico,

fibromialgia, poliomielite e catarata, respectivamente

Sem problemas associados

Fonte: Autores, Santa Maria, 2019.

Em relação a hábitos como fumar e beber, 51 mulheres relataram não fumar, três relatar fumar, uma relatou fumar eventualmente e uma mulher não informou. 43 mulheres relataram não beber, dez relataram beber eventualmente e três não informaram. Em relação ao hábito de praticar atividade física, 27 mulheres relataram não praticar, 21 relatam que praticam atividade física e oito mulheres não informaram. Das 56 mulheres atendidas, 40 relataram estar na menopausa, nove ainda estão na idade fértil e sete não informaram. No que tange à reposição hormonal, 40 mulheres relataram não fazer, sete relataram ter feito e nove mulheres não informaram. Das 56 mulheres, nove relataram menstruar.

Sobre os dados obstétricos, foi observada a média de 2,85 gestações por mulher. Os tipos de partos são apresentados na figura 1. Em relação à atividade sexual, 25 (44,64\%) relataram não ser ativa sexual, 24 (42,85\%) relataram ser ativa sexual e sete $(12,5 \%)$ não informaram.

Figura 1. Classificação do tipo de parto da amostra em porcentagem (\%).

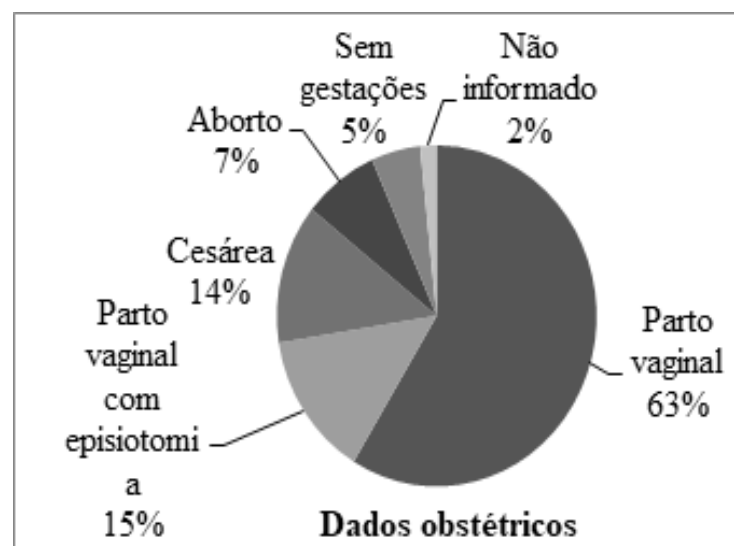

Fonte: Autores, Santa Maria, 2019. 
No que diz respeito aos hábitos urinários, foram elencados itens para uma maior compreensão e agrupamento dos dados. Nos hábitos urinários diurnos considerou-se como fisiológico de seis a oito vezes. Abaixo desse critério esteve presente em 22 mulheres, acima deste critério esteve presente em 25 mulheres e em nove mulheres não foi informado esses dados. Em relação aos hábitos urinários noturnos, 49 mulheres relataram ter hábitos noturnos. Ainda, sete mulheres não informaram sobre os seus hábitos. A tabela 2 descreve o tempo que a mulher apresentava os sintomas de IU bem como o caráter e o tipo de incontinência.

Tabela 2: Descrição do tempo de incontinência urinária, caráter e incontinência em número (n) e porcentagem (\%).

\begin{tabular}{|c|c|c|}
\hline \multicolumn{3}{|c|}{ Tempo de Incontinência urinária } \\
\hline & $\mathrm{n}$ & $\%$ \\
\hline Menos de um ano & 03 & $5,35 \%$ \\
\hline 1 a 3 anos & 14 & $25 \%$ \\
\hline 4 a 6 anos & 09 & $16,07 \%$ \\
\hline 7 a 10 anos & 03 & $5,35 \%$ \\
\hline Mais que 10 anos & 09 & $16,07 \%$ \\
\hline Não informado & 18 & $32,15 \%$ \\
\hline \multicolumn{3}{|c|}{ Caráter de IU } \\
\hline Jato & 31 & $49,20 \%$ \\
\hline Gota a gota & 11 & $17,46 \%$ \\
\hline Imperceptível & 03 & $4,76 \%$ \\
\hline Micção intermitente & 02 & $3,17 \%$ \\
\hline Sensação de queimação & 02 & $3,17 \%$ \\
\hline Ardência & 02 & $3,17 \%$ \\
\hline Perda ao esforço & 01 & $1,58 \%$ \\
\hline Dificuldade em segurar a urina & 01 & $1,58 \%$ \\
\hline Não informado & 10 & $15,87 \%$ \\
\hline \multicolumn{3}{|c|}{ Incontinência } \\
\hline Esforço & 28 & $35,44 \%$ \\
\hline Diurna & 17 & $21,51 \%$ \\
\hline Noturna & 13 & $16,45 \%$ \\
\hline Contínua & 06 & $7,59 \%$ \\
\hline Jato & 04 & $5,06 \%$ \\
\hline Gota a gota & 04 & $5,06 \%$ \\
\hline Urgência & 02 & $2,53 \%$ \\
\hline Não informado & 05 & $6,32 \%$ \\
\hline
\end{tabular}

Fonte: Autores, Santa Maria, 2019. 
Em relação ao uso e o tipo de proteção, os dados foram apresentados na figura 2 e 3, respectivamente. A tabela 3 apresenta a intensidade da perda de urina bem como os motivos contribuintes para essa perda. Já a tabela 4 apresenta os motivos da perda urinária da amostra.

Figura 2. Descrição do uso de proteção da amostra em número (n).

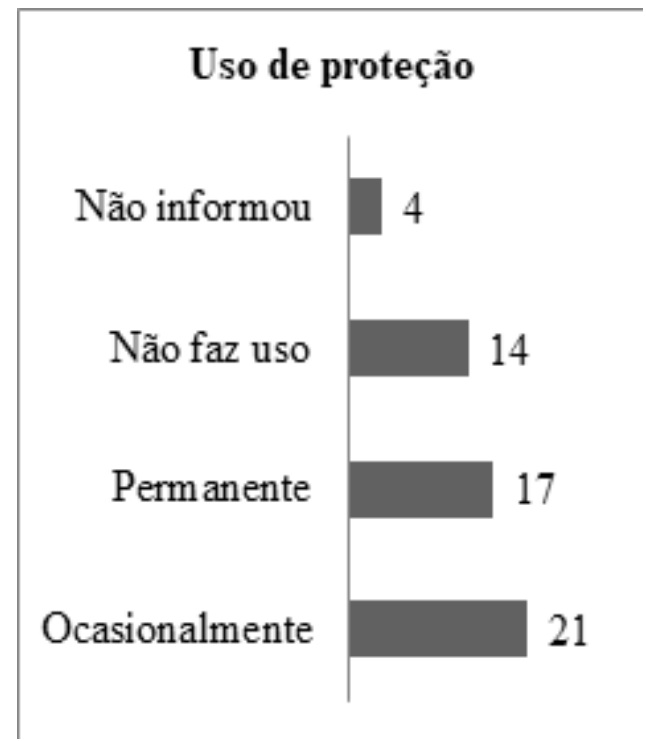

Fonte: Autores, Santa Maria, 2019.

Figura 3. Descrição do tipo de proteção da amostra em número (n).

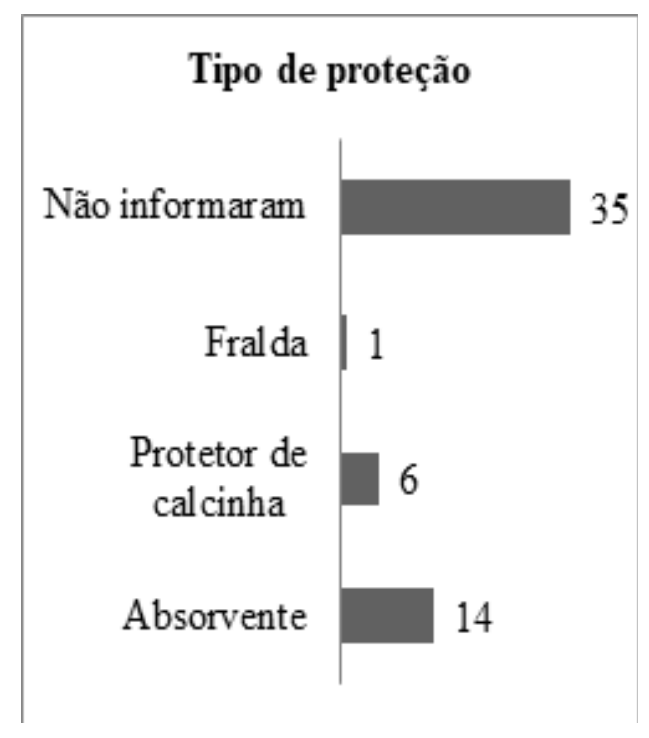

Fonte: Autores, Santa Maria, 2019. 
Tabela 3: Relação da intensidade da perda de urina e os motivos que contribuem para a perda ao esforço.

\begin{tabular}{lcc}
\hline & Intensidade de perda urinária de esforço & \\
\hline Mínima & 06 & $10,71 \%$ \\
Moderada & 07 & $12,5 \%$ \\
Intensa & 02 & $3,57 \%$ \\
Não informada & 41 & $73,21 \%$ \\
\hline \multicolumn{3}{c}{ Motivos que contribuem para a perda ao esforço } \\
\hline Tosse & 23 & $26,74 \%$ \\
Espirro & 17 & $19,76 \%$ \\
Esforço & 09 & $10,46 \%$ \\
Perda na corrida & 07 & $8,13 \%$ \\
Riso & 07 & $8,13 \%$ \\
Perda no salto & 05 & $5,81 \%$ \\
Perda no frio & 04 & $4,65 \%$ \\
Dança & 03 & $3,48 \%$ \\
Troca de posição & 03 & $3,48 \%$ \\
Relação sexual & 03 & $3,48 \%$ \\
Ginástica & 03 & $3,48 \%$ \\
Andar & 02 & $2,32 \%$ \\
Marcha rápida & 01 & $1,16 \%$ \\
Estar de cócoras & 01 & $1,16 \%$ \\
Stress & 01 & $1,16 \%$ \\
Urgência & 01 & $1,16 \%$ \\
Barulho de água & 01 & $1,16 \%$ \\
Mãos na água & 01 & $1,16 \%$ \\
\hline Fone:Autors Sala
\end{tabular}

Fonte: Autores, Santa Maria, 2019.

Tabela 4: Motivos de perda urinária na amostra

\begin{tabular}{lcc}
\hline & Motivos de perda urinária & \\
\hline Frio & 17 & $12,78 \%$ \\
Tosse & 14 & $10,52 \%$ \\
Espirro & 10 & $7,51 \%$ \\
Stress & 08 & $6,01 \%$ \\
Mãos na água & 08 & $6,01 \%$ \\
Andar & 08 & $6,01 \%$ \\
Riso & 08 & $6,01 \%$ \\
Barulho de água & 07 & $5,26 \%$ \\
Salto & 05 & $3,75 \%$ \\
Corrida & 05 & $3,75 \%$ \\
Troca de posição & 05 & $3,75 \%$ \\
Ginástica & 04 & $3,01 \%$ \\
Água corrente & 02 & $1,50 \%$ \\
Atividade física & 01 & $0,75 \%$ \\
Dança & 01 & $0,75 \%$ \\
Escovar os dentes & 01 & $0,75 \%$ \\
Não informaram & 29 & $21,80 \%$ \\
\hline
\end{tabular}

Fonte: Autores, Santa Maria, 2019. 


\section{DISCUSSÃO}

Com o envelhecimento, o trato urinário apresenta alterações como a diminuição da força de contração e atrofia da musculatura. Ainda, a capacidade vesical diminui e a perda de urina se torna comum devido à perda de elasticidade e contratilidade da bexiga. Também, pode se dar devido a alterações do sistema circulatório e nervoso ${ }^{5}$.

Neste estudo, a média da idade das pacientes atendidas no ambulatório de fisioterapia foi de 58,2 anos. Isso pode ser justificado pois com o envelhecimento estes sintomas são mais frequentes. A idade está relacionada com a perda de urina devido às modificações que ocorrem na função e na estrutura do sistema urinário e com o comprometimento da independência funcional ${ }^{6}$. Além disso, as alterações hormonais que surgem com o avanço da idade desencadeiam uma diminuição do nível de estrogênio que está presente na uretra, bexiga, vagina e na musculatura do assoalho pélvico, comprometendo a função do trato urinário inferior?

Quanto aos problemas associados, os mais encontrados foram a hipertensão arterial sistêmica (HAS) e constipação. A HAS tem associação com a IU devido ao uso das medicações para a regularização da pressão arterial bem como de medicamentos diuréticos pois os mesmos aumentam o débito urinário ${ }^{8}$. A constipação tem associação com a IU e podendo favorecer o aparecimento da IU. A constipação é um aspecto que pode ser alterado com mudanças de comportamento com a adoção de hábitos alimentares saudáveis bem como a prática de atividade física? .

A prática da atividade física age positivamente no mecanismo da continência pois influencia na manutenção do peso corporal visto que o aumento de gordura pode causar elevação crônica da pressão intra-abdominal e enfraquecer as estruturas que dão o suporte para o assoalho pélvico ${ }^{9}$. Neste estudo, a maioria das mulheres entrevistadas não praticavam atividade física.

Em relação aos dados obstétricos, 62,84\% foram parto vaginal e 14,75\% foram partos vaginais com episiotomia. O risco de desenvolver IU após o parto vaginal é de 67 a 71\%, embora somente a gestação também seja considerada como um fator de risco para a incontinência urinária ${ }^{10}$. A episiotomia rotineira é outro fator de risco visto que afeta negativamente a força dos músculos do assoalho pélvico"11.

O mecanismo que esclarece a relação da IU com a gestação não está completamente esclarecido. A gestação pode estar relacionada com a IU pela sequência da tensão, do estiramento e do enfraquecimento dos tecidos que compõem o assoalho pélvico ${ }^{12}$. Ainda, o crescimento uterino aliado às mudanças endócrinas, tais como o incremento da progesterona, a diminuição da relaxina e do colágeno podem contribuir na diminuição da função esfincteriana e da força muscular de suporte ${ }^{13}$. Além disso, os tipos de parto podem determinar os diferentes graus de lesão muscular, nervosa e do tecido conjuntivo com a consequente perda involuntária de urina ${ }^{14}$.

Após o parto vaginal pode ocorrer a redução do tônus vaginal bem como da força e da resistência dos músculos 
que compõem o assoalho pélvico ${ }^{15}$. Ainda, pode ocorrer em virtude do trauma neuromuscular do assoalho pélvico e por estiramento ou compressão mecânica dos nervos pélvicos, além de ocorrer pela pressão e distensão do assoalho pélvico com a passagem da cabeça do recém-nascido durante o segundo estágio do parto ${ }^{14}$. A perda urinária pode ocorrer entre dois e doze meses após o parto persistindo por muitos anos e é independente do tipo de parto ${ }^{14}$.

No que tange a incontinência, a perda de urina por esforço foi a mais encontrada (28) neste estudo. Mulheres em idade reprodutiva e até meia idade apresentam com mais frequência a incontinência de esforço, a partir dos 50 anos a maior prevalência passa a ser de urgência ou mista ${ }^{16,17}$. Em relação aos motivos que fazem ter a perda de urina ao esforço destaca-se a tosse e os espirros. A tosse e o espirro elevam a pressão intra-abdominal, podem causar danos ao assoalho pélvico e, com isso, ser um dos fatores de risco para a incontinência urinária ${ }^{9}$.

Este estudo apresenta como contribuição para a prática clínica a investigação de motivos que podem ocasionar as perdas urinárias afim de preveni-las ou até evita-las. Ainda, mulheres que apresentam estas perdas podem apresentar vergonha de abordar sobre essa temática tanto com a família como com profissionais de saúde, buscando atendimento somente em casos mais graves. Como limitação deste estudo destaca-se a falta de uniformidade em relação a faixa etária o que poderia distinguir melhor os sintomas conforme a idade. Ainda, destaca-se que muitas mulheres podem apresentar vergonha de consultar com profissionais do sexo masculino o que pode ter influenciar nas respostas dos questionários.

\section{CONSIDERAÇÕES FINAIS}

A incontinência urinária é bastante encontrada em mulheres no processo de envelhecimento devido às alterações que o corpo apresenta com o avanço da idade. Neste estudo, a IU esteve presente em mulheres com uma média de idade de 58,2 anos, casadas, com hipertensão ou constipação associadas e que não praticavam atividade física. Ainda, o número alto de gestações e de parto vaginal aumentou o número de mulheres incontinentes.

Houve um número grande de mulheres que relatavam ter muitas perdas durante o dia e pouca perda a noite, em forma de jato, ao esforço e que ocorriam após a tosse ou espirros. Espera-se que com estes dados se tenha um maior conhecimento sobre a incontinência urinária e seus agravos e se possam elaborar ações para desmistifica-las.

\section{REFERÊNCIAS}

1. Santos MO. Influência da incontinência urinária na qualidade de vida de idosos [monografia]. Lagarto: Universidade Federal de Sergipe, Curso de Fisioterapia, Departamento de Fisioterapia; 2018. 
2. Padilha JF, Silva AC, Maso GZ, Marques CMG. Investigação da qualidade de vida de mulheres com incontinência urinária. Arq. Ciênc. Saúde UNIPAR 2018;22 (1):43-48.

3. Saboia DM, Firmino MLV, Bezerra KC, Neto JAV, Ória MOB, Vasconcelos CTM. Impacto dos tipos de incontinência urinária na qualidade de vida de mulheres. Rev. esc. enferm. USP 2017;51:e03266.

4. Silva LWS, Lucas TQC, Santos SSO, Novaes VS, Pires EPOR, Lodovici FMM. Fisioterapia na incontinência urinária: olhares sobre a qualidade de vida de mulheres idosas. Rev Kairos 2017;20 (1):221-238.

5. Quadros LB, Aguiar A, Menezes AV, Alves EF, Nery T, Bezerra PP. Prevalência da incontinência urinária em idosos institucionalizados e sua relação com o estado mental, independência funcional e comorbidades associadas. Acta Fisiatr. 2015;22 (3):130-134.

6. Silva VA, Souza KL, D’Elboux MJ. Incontinência urinária e os critérios de fragilidade em idosos em atendimento ambulatorial. Rev. esc. enferm. USP 2011;45 (3):672-678.

7. Sacomori C, Negri NB, Cardoso FL. Incontinência urinária em mulheres que buscam exame preventivo de câncer de colo uterino: fatores sociodemográficos e comportamentais. Cad. Saúde Pública 2013;29 (6):1251-1259.

8. Marques LP, Schneider IJC, Giehl MWC, Antes DL, D’Orsi E. Condições demográficas, condições de saúde e fatores de estilo de vida associados à incontinência urinária em idosos de Florianópolis, Santa Catarina, Brasil. Rev Bras Epidemiol. 2015; 18(3): 595-606.

9. Oliveira TM, Valdez FML, Lima KES, Magalhães MS, Abdon APV, Bezerra IN. Prevalência de incontinência urinária e fatores associados em mulheres no climatério em uma unidade de atenção primária à saúde. Rev Bras Promoç Saúde 2015;28 (4):606-612.

10. Gyhagen M, Bullarbo M, Nielsen TF, Milson I. The prevalence of urinary incontinence 20 years after childbirth: a national cohort study in singleton primiparae after vaginal or caesarean delivery. BJOG. 2013;120 (2):144- 51.

11. Carvalho CCM, Souza ASR, Moraes Filho OB. Episiotomia seletiva: avanços baseados em evidências. 
Femina 2010;38 (5):265-70.

12. Hernández RRV, Aranda ER, Aznar CT. Urinary incontinence and weight changes during pregnancy and post partum: Apending challenge. Midwifery. 2013;29:123- 129.

13. Sangsawang B. Risk factors for the development of stress urinary incontinence during pregnancy in primigravidae: a review of the literature. Eur J Obstet Gynecol Reprod Biol. 2014;178:27-34.

14. Dellu MC. Incontinência urinária no climatério: prevalência, fatores associados e impacto na qualidade de vida [Tese]. São Paulo: Universidade de São Paulo, Programa de Pós Graduação em Saúde Pública; 2015.

15. Hilde G, Staer-Jensen J, Siafarikas F, Engh ME, Brækken IH, Bø K. Impact of childbirth and mode of delivery on vaginal resting pressure and on pelvic floor muscle strength and endurance. Am J Obstet Gynecol. 2013;208:50.17.

16. Abrams P, Anderson KE, Birder L, Brubaker L, Cardoso L, Chapple C, et al. Fourth International Consultation on Incontinence Recommendations of the International Scientific Committee: evaluation and treatment of urinary incontinence, pelvic organ prolapse, and fecal incontinence. Neurourol Urodyn. 2010;29 (1):213-40. 